\title{
The Triad Of PCOS, Infertility And Metformin
}

\author{
Ayesha Khan, Nasim Karim, Jahan Ara Ainuddin
}

\section{ABSTRACT:}

Polycystic ovarian syndrome (PCOS) is now considered as a complicated disease of endocrine system. It is found in 5$10 \%$ of women of fertile age group. It was initially considered as a reproductive disorder in women. Studies in recent past have documented that PCOS is a metabolic syndrome and is influenced by genetic and environmental factors. It is also transferred genetically, an autosomal dominant trait with variable penetrance. Since role of insulin resistance has been identified as a main culprit in the development of PCOS therefore use of insulin sensitizers to treat PCOS seems logical. Insulin sensitizer such as metformin is a therapeutic option that targets not only insulin resistance present in this syndrome but several other aspects especially reproductive abnormalities such as infertility, which is a major problem of concern for women of reproductive age. This review attempts to cover the use of metformin in PCOS infertile women.

Key Words:: Polycystic ovarian syndrome, Reproductive abnormalities, Obesity, Insulin resistance, Infertility, Metformin

\section{INTRODUCTION:}

Polycystic ovarian syndrome (PCOS) is a metabolic syndrome that occurs in one out of seven women of reproductive age. ${ }^{1}$ It is prevalent in $5-10 \%$ of women ${ }^{2}$. More than half of patients with PCOS have increased basal metabolic rate or are obese and are therefore at increased risk to develop obesity-related concomitant diseases ${ }^{3}$. PCOS is influenced by genetic and environmental factors too $0^{4,5}$. Pathophysiology of PCOS is multifocal either due to continuously rapid gonadotropin-releasing hormone pulses, hypersecretion of luteinizing hormone and inadequate secretion of follicle-stimulating hormone all take part to excessive production of ovarian androgen and disturbances in ovarian functions ${ }^{6}$. Insulin resistance is the main factor in development of PCOS. Insulin increases adrenal and ovarian steroidogenesis and inhibits liver synthesis of sex hormone-binding globulin ${ }^{7}$. In 2003, the Rotterdam Consensus Conference proposed that PCOS can be diagnosed, after the exclusion of other endocrine diseases, by at least two out of three following criteria: (1) clinical or biochemical hyperandrogenism, (2) oligomenorrhea or anovulation, and (3) polycystic ovary morphology ${ }^{8}$.

PCOS is considered to begin during the pubescent ${ }^{9,10}$. In the first two years of life disturbances occur in menstrual cycle and ovulation during the start of menstruation (Twenty-five percent of girls in the first year of life, thirty- forty percent

Г Ayesha Khan - - - - - - - - - ᄀ I Senior Lecturer, M.Phil Student, Pharmacology Department Bahria University Medical \& Dental College

I Email: dr.ayeshakhan85@gmail.com

I Nasim Karim

I Professor \& HOD

I Pharmacology Department

Bahria University Medical \& Dental College

I Jahan Ara Ainnuddin

Professor Obstetrics \& Gynecology

I Dow International Medical College (DIMC)

I Dow University of Health Sciences (DUHS), Karachi

I Received: 25-10-18

I Accepted: 07-12-18 $--------\ldots-1$ up to the third and fourth year). Complete pubertal development usually occurs in the first five years after the onset of menstrual cycle. Menstrual cycle varies from one female to another female, persistence of oligomenorrhea (menstrual cycles more than thirty five days), secondary amenorrhea (absence of menstrual cycles for more than three months) or primary amenorrhea, absence of onset of menstruation at 16 years of age ${ }^{11}$ in girls with complete development of secondary sexual characteristics and good adult height, which indicate an excess of androgens levels in the blood ${ }^{12}$. Long duration of oligomenorrhea during pubescent at 14-19 years of age is indicated as the signs of persistent ovarian dysfunction in the future ${ }^{13}$. Acne on the center of the face and on the forehead is very common which is usually present during the adolescence period with increasing prevalence from 15 to 20 years of age, sometimes it become aggressive which also indicate sign of increased androgen levels in $\operatorname{PCOS}^{14,15}$. Alopecia is not considered as a common finding during pubescent, so it is not a common indicator of hyperandrogenism to rule out clinically. However in $65 \%$ of the patients during the adolescence period, hirsutism may be a good predictor of $\mathrm{PCOS}^{16}$.

During the reproductive age group, it may cause failure in ovulation which is associated with increased risk of complications during pregnancy, such as abortions, gestational diabetes and preeclampsia ${ }^{17}$. Infertility and hirsutism are the two problematic conditions in a woman with PCOS that bring the women to the hospital. If we talk about infertility, there is hyperandrogenemia and excessive secretion of luteinizing hormone that can lead to menstrual disturbances, features of hyperandrogenemia and decreased fertility rate. Disturbances in menstrual cycles, such as oligomenorrhea or amenorrhea, are the initial feature of anovulation that is usually noticed by the doctor during the visit. Usually, these women will have increased number of small antral follicles on ultrasound and raised serum anti-mullerian hormone $(\mathrm{AMH})$ concentration $^{18}$. Insulin resistance, associated hyperinsulinemia, increased body mass index and obesity are significant issues of polycystic ovarian syndrome ${ }^{19}$. 
These factors may play an important role in the pathogenesis of androgen excess and the susceptibility to develop earlier than expected glucose intolerance states and type 2 diabetes. Decreased insulin sensitivity causes the differentiation of pre-adipocytes to adipocytes, especially in the region of abdomen, which facilitate the deposition of fat and causes visceral-type obesity in women with $\mathrm{PCOS}^{20}$.

Polycystic ovarian morphology can be detected on histopathology however clinically large number of cases of polycystic ovarian morphology are detected by transvaginal ultrasonography. Polycystic ovarian morphology is defined as at least one ovary and greater than $10 \mathrm{~cm}^{3}$ ovarian volume (or $10 \mathrm{~mL}$ ) or an increased number of antral follicles (i.e. those that can be visualized as cysts in the ovarian cortex measuring $2-9 \mathrm{~mm}$ in diameter). The exact number of antral follicles, that is the antral follicle count to establish the diagnosis of polycystic ovarian morphology using modern high frequency transvaginal ultrasonography probes is now at least 18 if not higher ${ }^{21}$.

The first and most effective treatment of PCOS is life-style modification and weight loss ${ }^{22}$. Hormonal contraceptives are commonly used to reduce hyperandrogenic symptoms such as acne and hirsutism and to normalize the menstrual cycle $^{23}$. In recent years' insulin-sensitizing drugs have been widely prescribed for PCOS patients. These drugs include Metformin, Pioglitazone, D-chiro-inositol. Metformin, a biguanide is proven to be effective not only for insulin resistance and body weight reduction but also for menstrual irregularity, anovulatory cycles, signs of hyperandrogenism and gestational complications ${ }^{24}$. Administration of metformin causes significant reduction in weight and successful ovulation in PCOS patients, within the time period of 3 months after start of treatment. Thus metformin produces spontaneous ovulation and better outcomes ${ }^{25}$.

\section{METHODOLOGY:}

We used search engines of Google, Google scholar and Pubmed for searching literature. Key words and phrases used were PCOS, Insulin resistance, hyperinsulinemia, hyperandrogenemia, hirsutism, acne, infertility, obesity, polycystic ovarian syndrome, reproductive and cardiovascular abnormalities, cyclical problems, anovulation and use of insulin sensitizers.

Filter used was search period 2012-2018.Total 89 articles were selected for writing the review. Out of these 36 articles were non-relevant on account of the use of oral contraceptives, clomiphene citrate and insulin sensitizer other than metformin. Three articles were in non- English language while 6 studies were on rabbits and mice (non-human). Finally, 44 articles are included for write up of this review article.

\section{LITERATURE REVIEW:}

In 1935 Stein and Leventhal explained the term polycystic ovarian syndrome, also called as syndrome X or Stein-
Leventhal syndrome. PCOS is characterized as the major cause of WHO classified type - 2 anovulatory infertility. Polycystic ovarian syndrome mostly present in women of reproductive age ${ }^{26}$. Features of polycystic ovarian syndrome are irregular period or amenorrhea, increased hair growth and infertility. Fifty to seventy percent of women with polycystic ovarian syndrome are affected by Insulin resistance that can lead to metabolic syndrome, hypertension, dyslipidemia, alteration in the level of glucose, and diabetes. There is also increased risk of deposition of calcium in coronary artery. Depression, anxiety and bipolar disorder are commonly found in PCOS. Environmental and genetic history origin both are considered as an etiological factor. Irregular menstruation is common feature of PCOS but $30 \%$ have normal menses, $30 \%-40 \%$ have amenorrhea and $85 \%-90 \%$ have oligomenorrhea ${ }^{27}$.

Genome wide association studies (GWAS) has documented that SNP (rs11031006) related to PCOS resides in the region of follicle-stimulating hormone (FSH)-b polypeptide (FSHB) and ARL14EP gene which is strongly associated with luteinizing hormone ${ }^{28}$.

\section{PATHOPHYSIOLOGY OF PCOS:}

Insulin resistance plays a main role in the pathogenesis of PCOS. Decreased sensitivity of insulin is aggravated by weight gain, which in turn exacerbates the clinical, reproductive, and metabolic features of the condition. It can lead to hyperinsulinaemia and subsequent abnormal follicular development, which in turn causes elevation of estrogen and androgen levels. Sex hormone-binding globulin decreases due to hyperinsulinaemia and the systemic effects are exacerbated by a reduction in sex hormone binding globulin (SHBG) along with persistently elevated luteinizing hormone (LH) levels, which arrest ovulation. High levels of circulating free androgens are responsible for the clinical features associated with PCOS, and the absence of ovulation causes unopposed estrogen stimulation on the endometrium and associated hyperplasia with resultant infertility ${ }^{29}$.

\section{DIAGNOSIS OF PCOS:}

Anovulatory infertility due to PCOS is diagnosed upon

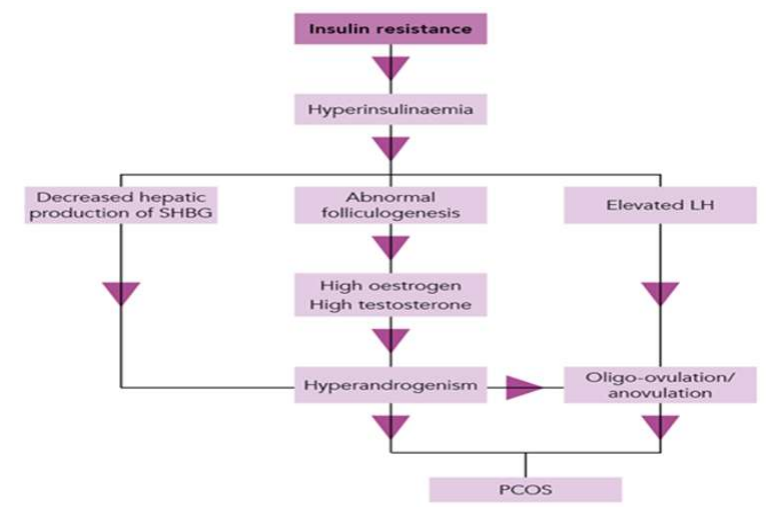

Firure 1. Hormonal changes that influence the development of PCOS 
presence of two out of three features (a) evidence of hyperandrogenemia clinically and/or biochemically (b) oligo-ovulatory or anovulatory cycles (c) presence of more than 12 follicles in each ovary measuring $29 \mathrm{~mm}$ and/or increased ovarian volume $(>10 \mathrm{~mL})$ on ultrasonography. ${ }^{8}$ Diagnosis of PCOS in anovulatory women is confirmed by a hormonal profile including serum levels of testosterone (or androstenedione) and gonadotropins - increased level of luteinizing hormone and normal level of follicle stimulating hormone, adds weight to the diagnosis of disease. Additionally, Serum prolactin level and the estimation of estrogen status in patients with amenorrhoea is also required. Routine thyroid function tests are also considered in PCOS. The importance of screening for autoimmune thyroid disease is underlined by the association of primary hypothyroidism with an increased risk of hyper-prolactinaemia and primary ovarian insufficiency ${ }^{30}$. Estimation of anti-mullerian hormone, as a new diagnostic tool (made by antral follicles, which are increased during polycystic ovaries) in combination with luteinizing hormone levels has high sensitivity and specificity for the diagnosis of polycystic ovary syndrome ${ }^{31}$.

\section{MANAGEMENT OF PCOS:}

Lifestyle modification (LSM) is considered to be the first step of management. It recommends that overweight patients with PCOS should control their diet that is to consume $1200-1500 \mathrm{kcal} /$ day and ensure that they should engage in moderate exercise for at least $30 \mathrm{~min}$ per day for at least five days a week. By restricting caloric intake, engaging in exercise, reducing BMI addressing behavior, receiving metformin, giving up smoking and drinking, patients can improve insulin resistance and free testosterone levels as well as reduce menstrual disorders, excess hair growth, acne and above all restore fertility ${ }^{32}$.

As mentioned earlier insulin sensitizers are the mainstay of treating PCOS related infertility as underlying pathology revolves around insulin resistance. Among the insulin sensitizers metformin is a well-tested or it may not be wrong if the term conventional insulin sensitizer is used for this drug as it is in use for PCOS since 1994. Metformin is a guanidine derivative that was obtained from the plant Galega officinalis (French lilac) at first time and has been used for more than 60 years to reduce hyperglycemia ${ }^{33}$. Metformin decrease the fasting levels of plasma insulin, C-peptide and proinsulin-like molecules. It also increases insulin binding, enhancing the utilization of glucose peripherally, and reduces the formation of glucose by the liver. Androgen production by theca cell also reduces significantly in vitro ${ }^{34}$. Metformin has a positive effect on ovulation and also stimulates $\mathrm{it}^{35}$. It reduces the risk of ovarian hyperstimulation syndrome for IVF (in vitro fertilization) during controlled ovarian stimulation before or during treatment with metformin is another advantageous option for the patients ${ }^{36}$. Metformin is a class B drug in the US food and drug administration's drug safety classification, although a meta-analysis have showed that early use of metformin by PCOS patients did not increased the risk of birth defects ${ }^{37}$.

\section{PHARMACOKINETICS OF METFORMIN:}

Metformin is taken by oral route and excreted unchanged in the urine. Its half-life is almost equal to five hours. The drug is well distributed in all body tissues including the intestine, liver, and kidney by organic cation transporters. It is prescribed $1500-2500 \mathrm{mg}$ daily in two or three divided doses. Metformin is well tolerated but in some patients it may cause nausea, vomiting, diarrhea and bloating. This usually settles as the treatment continues. However, escalation dose therapy not only prevents these features but also improves patient's compliance to treatment. Physician should inform the patients about the gastrointestinal side effects of the drug before starting the treatment that last for two to three weeks. Patients should be advised to take the drug with meals to avoid the side effects and dose should be gradually increased to improve the compliance ${ }^{38}$.

\section{PHARACODYNAMICS OF METFORMIN IN PCOS INDUCED INFERTILITY:}

Metformin's primary action is to inhibit the production of hepatic glucose, decrease lipid synthesis, increase fatty acid oxidation and inhibit gluconeogenesis resulting in a decrease in circulating insulin and glucose $e^{3}$. It has been proved that metformin enhances the ability of insulin to induce GLUT4 translocation to the plasma membrane. Metformin activates AMP-kinase enzyme also ${ }^{40}$. In PCOS, metformin increases insulin release and inhibits androgenic excess via effects on steroidogenic acute regulatory protein and 17 alphahydroxylase. Metformin improves insulin resistance and compensatory hyperinsulinemia associated with PCOS. It increases production of Sex hormone-binding globulin (SHBG) and reduces androgens in circulation. This in turn improves the function of theca and granulosa cells of the ovaries that increases fertility. Metformin also exerts pleiotropic effects on insulin sensitive tissue such as liver, skeletal muscles, adipose tissue, endothelium and ovaries. In addition, it also has a direct effect on the ovaries and improves cyclical disturbances and ovulation rates in most of the PCOS women. Moreover, Gonadotropin-releasing hormone $(\mathrm{GnRH})$ neurons in hypothalamus regulate fertility through central mechanism which is dependent on $5^{\prime}$ adenosine monophosphate-activated protein kinase (AMPK) pathway. Metformin inhibits 5' adenosine monophosphateactivated protein kinase (AMPK) pathway as well as neuropeptide $-Y$ neurons in the hypothalamus and thereby improves fertility and produces suppression of appetite in $\operatorname{PCOS}^{41}$.

\section{ADVERSE EFFECTS AND CONTRAINDICATIONS OF METFORMIN:}

Diarrhea, nausea and or abdominal discomfort are the most common side effects associated with metformin. These symptoms occur in $50 \%$ of patients after taking the drug. 
They are mild and usually settle as the treatment is continued or at low doses. It produces side effects in five percent of the patients, even at low doses. When the drug accumulates in the enterocytes of the small intestine these types of side effects may be produce. Slow-release or extended release formulations are associated with less gastrointestinal upset ${ }^{42}$. A dangerous side effect of Metformin is lactic acidosis through interference with mitochondrial respiration because of this blood lactate levels rises as there is conversion of glucose to lactate. This is also caused by toxic range of drug level in blood (> $5.0 \mathrm{mg} / \mathrm{l}$ ) [normal range, $0.5-2.0 \mathrm{mg} / \mathrm{l}^{43}$. Use of Metformin for prolong period of time interfere with the absorption of vitamin B12 level therefore it is recommended that level of vitamin $\mathrm{B}_{12}$ should be monitored in anemic patients or in patients with peripheral neuropathy. Therefore, oral formulation of vitamin $\mathrm{B}_{12}$ should be given to the patients on long term use. Before the use of Metformin, physician should inform the patient about the side effects of the drugs ${ }^{44}$. Use of Metformin should be avoided in many situations that increases the risk of lactic acidosis, such as severe dehydration, infection, shock, heart failure, recent myocardial infarction, severe peripheral vascular disease, hepatic impairment, alcohol dependency.

\section{CONCLUSIONS:}

Metformin has multiple beneficial effects in the treatment of PCOS. It helps in reduction of weight and restoration of menses with normal functions of the ovaries. It also improves hyperinsulinemia and hyperandrogenemia associated with PCOS induced infertility. Metformin increases the rate of ovulation and conception in women with PCOS and hence represents the apex of the triad with the base being formed by PCOS and infertility. In other words, the base of PCOS and infertility converge towards the apex of solution which is metformin.

\section{REFERENCES:}

1. Bozdag G, Mumusoglu S, Zengin D, Karabulut E, Yildiz BO. The prevalence and phenotypic features of polycystic ovary syndrome: a systematic review and meta-analysis. Hum Reprod. 2016;31(12):2841-55.

2. Escobar-Morreale HF. Polycystic ovary syndrome: definition, aetiology, diagnosis and treatment. Nat Rev Endocrinol. 2018;14(5):270-84.

3. Kim JY, Tfayli H, Michaliszyn SF. Distinguishing characteristics of metabolically healthy versus metabolically unhealthy obese adolescent girls with polycystic ovary syndrome. FertilSteril. 2016; 105:1603-11.

4. Rosenfield RL. The diagnosis of polycystic ovary syndrome in adolescents. Pediatrics. 2015; 136:1154-65.

5. Kosova G, Uebanek M. Genetics of the polycystic ovary syndrome. Mol Cell Endocrinol. 2013; 373:29-38.

6. McCartney CR, Marshall JC. Polycystic ovary syndrome. N Engl J Med. 2016; 375:54-64.

7. De Leo V, Musacchio MC, Cappelli V. Genetic, hormonal and metabolic aspects of PCOS: an update. Reprod Biol Endocrinol. 2016; 14:38.
8. Rotterdam ESHRE/ASRM-Sponsored PCOS Consensus Workshop Group. Revised 2003 consensus on diagnostic criteria and long-term health risks related to polycystic ovary syndrome. FertilSteril. 2004; 81(1):19-25.

9. Manmohan K. Kamboj, Andrea E. Bonny. Polycystic ovary syndrome in adolescence: diagnostic and therapeutic strategies 2017; 6(4): 248-255. doi:10.21037/tp.2017.09.11.

10. Ibanez L, Ong KK, Lopez-Bermejo A. Hyperinsulinaemic androgen excess in adolescent girls. Nat Rev Endocrinol. 2014;10 (8):499-508.

11. Marsh CA, Grimstad FW. Primary amenorrhea: diagnosis and management. Obstet Gynecol Surv. 2014;69:603-12.

12. Witchel SF, Oberfield S, Rosenfield RL. The diagnosis of polycystic ovary syndrome during adolescence. Horm Res Paediatr. 2015;87:376-89.

13. Glueck CJ,Woo JG, Khoury PR, Morrison JA, Daniels SR, Wang P. Adolescent oligomenorrhea (age 14-19) tracks into the third decade of life (age 20-28) and predicts increased cardiovascular risk factors and metabolic syndrome. Metabolism. 2015;64:539- 53.

14. Shen Y, Wang T, Zhou C. Prevalence of acne vulgaris in Chinese adolescents and adults: A community-based study of 17,345 subjects in six cities. Acta Derm. Venereol. 2012; 92: 40-4.

15. Eichenfield LF, Krakowski AC, Piggott C. Evidence-based recommendations for the diagnosis and treatment of pediatric acne. Pediatrics. 2013; 131 (Suppl. 3): S163-86.

16. Roe AH, Prochaska E, Smith M. Using the androgen excessPCOS society criteria to diagnose polycystic ovary syndrome and the risk of metabolic syndrome in adolescents. J Pediatr. 2013;162(5):937-41.

17. Maliqueo M, Lara HE, Sanchez F. Placental steroidogenesis in pregnant women with polycystic ovary syndrome. Eur J ObstetGynecolReprodBiol. 2013;166:151-5.

18. Jose Bellver et al;Polycystic ovary syndrome throughout a woman's life.J Assist Reprod Genet. 2018; 35:25-39. doi: $10.1007 / \mathrm{s} 10815-017-1047-7$

19. Diamanti-Kandarakis E, Dunaif A. Insulin resistance and the polycystic ovary syndrome 1093 revisited: an update on mechanisms and implications. Endocrine Reviews 2012; 33: 981-1094. doi: 10.1210/er.2011-1034.

20. Barbosa-Desongles A, Hernández C, Simo R,Selva DM. Testosterone induces cell proliferation and cell cycle gene overexpression in humanvisceralpreadipocytes. Am J Physiol Cell Physiol. 2013; 305: C355-59.

21. Dewailly D, Lujan ME, Carmina E, Cedars MI, Laven J, Norman RJ, et al. Definition and significance of polycystic ovarian morphology: a task force report from the Androgen Excess and Polycystic Ovary Syndrome Society. Hum Reprod Update. 2014; 20:334-52.

22. Balen AH, Morley LC, Misso M. The management of anovulatory infertility in women with polycystic ovary syndrome: an analysis of the evidence to support the development of global WHO guidance. Hum Reprod Update. 2016; 22:687-708.

23. Mendoza N, Simoncini T, Genazzani AD. Hormonal contraceptive choice for women with PCOS: a systematic review of randomized trials and observational studies. Gynecol Endocrinol. 2014;25:1-11.

24. $\mathrm{Hu} \mathrm{L}$, Shen $\mathrm{H}, \mathrm{Wu} \mathrm{QF}$. Treatment of polycystic ovarian 
syndrome with insulin resistance by insulin-sensitizer. Clin Exp Obstet Gynecol. 2014;41:288-92.

25. Panidis D, Tyiomalos K, Papadakis E, Katsikis I. Infertility treatment in polycystic ovary syndrome: lifestyle interventions, medications and surgery. Front Horm Res. 2013; 40: 128141.

26. Hashim H A. Twenty years of ovulation induction withmetformin for PCOS; what is the best available evidence? Reproductive BioMedicine Online.2016; 32: 44-53.

27. Sirmans SM, Pate KA. Epidemiology, diagnosis and management of polycystic ovary syndrome.Clin Epidemiol. 2014; 6:1-13. doi:10.2147/CLEP.S37559.

28. Day FR, Hinds DA, Tung JY, et al. Causal mechanisms and balancing selection inferred from genetic associations with polycystic ovary syndrome. Nat Commun. 2015;6:8464.

29. Connolly A, Beckett V. Polycystic ovary syndromemanagement of a long-term condition in primary care. In: Connolly A, Britton A, editors. Women's health in primary care. Cambridge: Cambridge University Press, 2017: 111-18.

30. Jayasena, C N, Franks S. The management of patients with polycystic ovary syndrome. Nature Reviews Endocrinology, 2014;10 (10), 624-36. doi:10.1038/nrendo.2014.102

31. Homburg R, Ray A, Bhide P. The relationship of serum antiMullerian hormone and polycystic ovarian morphology and polycystic ovary syndrome: a prospective cohort study. Human Reprod. 2013; 28:1077-83.

32. Panidis D, Tziomalos K, Papadakis E. Lifestyle intervention and anti-obesity therapies in the polycystic ovary syndrome: impact on metabolism and fertility.Endocrine. 2013;44:58390.

33. Sanchez-Rangel, E. Inzucchi SE. Metformin: Clinical use in type2 diabetes. Diabetologia. 2017; 60:1586-93.

34. Sivalingam VN, Myers J, Nicholas S, Balen AH, Crosbie EJ. Metformin in reproductive health, pregnancy and gynaecological cancer: established and emerging indications. Hum Reprod Update. 2014;20:853-68.
35. Morley LC, Tang T, Yasmin E, Norman RJ, Balen AH. Insulinsensitizing drugs (metformin, rosiglitazone, pioglitazone, Dchiro-inositol) for women with polycystic ovary syndrome, oligo amenorrhoea and subfertility. Cochrane Database Syst Rev. 2017 Nov 29;11: CD003053.

36. Tso LO, Costello MF, Albuquerque LE, Andriolo RB, Macedo CR. Metformin treatment before and during IVF or ICSI in women with polycystic ovary syndrome. Cochrane Database Syst Rev.2014 ;11: CD006105.

37. Cassina M, Dona M, Di Gianantonio E. First-trimester exposure to metformin and risk of birth defects: a systematic review and metaanalysis. Hum Reprod Update. 2014; 20:656-69.

38. Li Gong, Goswami S, Giacomini KM, Altman RB, Klein TE. Metformin pathways: pharmacokinetics and pharmacodynamics. Pharmacogenet Genomics. 2012 ; 22(11): 820-27. doi:10.1097/FPC.0b013e3283559b22.

39. Rena G, Pearson ER, Sakamoto K. Molecular mechanism of action of metformin: old or new insights? Diabetologia. 2013;56:1898-906.

40. Naderpoor N, Shorakae S, Courten B, Misso M L, Moran L J, Teede H J. Metformin and lifestyle modification in polycystic ovary syndrome: systematic review and metaanalysis. Human Reproduction Update. 2015; 21(5): 560-74. doi:10.1093/humupd/dmv025.

41. Pasquali R.Contemporary approaches to the management of polycystic ovary syndrome. Ther Adv EndocrinolMetab. 2018;9 (4)123-34. doi: 10.1177/2042018818756790.

42. Elizabeth Sanchez-Rangel1, Silvio E, Inzucchi. Metformin: clinical use in type 2 diabetes Diabetologia. 2017;60:1586 93 doi:10.1007/s00125-017-4336-x.

43. DeFronzo R, Fleming GA, Chen K, Bicsak TA.Metformin associated lactic acidosis: current perspectives on causes and risk. Metabolism. 2016; 65:20-29.

44. Aroda VR, Edelstein SL, Goldberg RB. Long-term metformin use and vitamin B12 deficiency in the Diabetes Prevention Program Outcomes Study. J Clin Endocrinol Metab. 2016;101:1754-61. 